\title{
Lack of Association of LPA Gene Polymorphisms with Coronary Artery Disease in Pakistani Subjects
}

\author{
Saleem Ullah Shahid, ${ }^{1}$ Shabana N A $\mathbb{D},{ }^{1}$ and Steve Humphries ${ }^{2}$ \\ ${ }^{1}$ Institute of Microbiology and Molecular Genetics, University of the Punjab, Lahore, Pakistan \\ ${ }^{2}$ Institute of Cardiovascular Sciences, University College London, UK \\ Correspondence should be addressed to Shabana N A; shabana.mmg@pu.edu.pk
}

Received 1 March 2021; Revised 27 May 2021; Accepted 8 June 2021; Published 12 June 2021

Academic Editor: Gaetano Isola

Copyright ( 2021 Saleem Ullah Shahid et al. This is an open access article distributed under the Creative Commons Attribution License, which permits unrestricted use, distribution, and reproduction in any medium, provided the original work is properly cited.

\begin{abstract}
Coronary artery disease (CAD) is the leading cause of death worldwide. Pakistan faces a high epidemic of CAD, and the disease burden is increasing with the passage of time. Several genetic markers have been reported to be significantly associated with CAD; one of them is the lipoprotein A gene. The aim of the current investigation was to genotype the LPA gene SNPs, rs3798220 and rs10455872, in Pakistani subjects with CAD in a case control study design. The genotyping was done by TaqMan allelic discrimination assay. The results showed that the cases had significantly higher prevalence of diabetes (64.6\%), hypertension (62.1\%), and smoking habits (29.5\%). The level of cholesterol in cases was higher than in controls $(208.25 \pm 54.11$ vs. $175.34 \pm 43.51, p \leq 0.0001)$. The LDL-C was higher in cases than in controls $(104.62 \pm 37.94$ vs. $77.05 \pm 21.17, p \leq 0.0001)$. Similarly, triglycerides were also higher in cases than in controls $(214.51 \pm 74.60$ vs. $190.54 \pm 70.26, p \leq 0.0001)$, whereas HDL-C was lower in cases than in controls $(45.13 \pm 11.63$ vs. $67.9 \pm 17.57, p \leq 0.0001)$. For rs3798220, the risk allele (C) frequency was 0.005 in cases and 0.002 in controls. For rs10455872, the risk allele (G) frequency was 0.017 in cases and 0.014 in controls. The risk allele frequencies were not significantly different between cases and controls $(p>0.05)$. In conclusion, these two LPA SNPs do not contribute significantly to CAD progression and cannot be used as independent risk factors for CAD in Pakistani population.
\end{abstract}

\section{Introduction}

Coronary artery disease (CAD) is a complex polygenic disease affecting individuals globally. A number of risk factors (modifiable and nonmodifiable) predispose to CAD [1]. Dietary habits, physical activity levels, genetic predisposition, and general health status interact to affect the outcome. In the recent years, many salivary mediators (e.g., galectin-3, interleukin-6, and urokinase-type plasminogen activator receptor) of $\mathrm{CAD}$ have been identified that clearly demonstrate the role of gingival health and other salivary components in the endothelial function [2-4].

Lipoprotein(a), LP(a), is a complex molecule consisting of an apolipoprotein, apo(a) attached to LDL molecule. The apo(a) of LP(a) is bound to LDL moiety at apolipoprotein B-100 (apoB-100) through a disulfide linkage [5]. The apo(a) is synthesized in the liver and then assembles with apoB-100 of LDL [6]. LP(a) inhibits plasminogen activation promoting thrombus formation [7]. It participates in the development of CAD through complex proatherogenic and prothrombotic pathways [8]. By accumulating in subendothelium, LP(a) accelerates cholesterol deposition, increased production of adhesion compounds, and aggregation of monocytes [9]. It can also bind to oxidized phospholipids mediating inflammation [10]. LP(a) thus can promote thrombosis either directly or by attenuating the thrombolysis.

The LPA gene (OMIM number 152200) is considered to be evolved from the plasminogen gene. The plasminogen gene with its 5 types of Kringle (K) domains (KI-KV) and a protease-like domain was first duplicated and then modified extensively to give rise the human LPA gene. During this remodelling process, the KI, KII, and KIII domains were lost while KV has retained a single copy. The protease-like domain changed and reshuffled so critically that it lost 
plasmin activity [11-13]. In the LPA gene, the KIV has been duplicated to KIV 1-10 and its copy number ranges from 2 to more than 40 . This KIV-2 copy number polymorphism is responsible for variations in circulatory levels of LP(a) [14]. The LP(a) concentrations also vary among healthy people of different ancestries [15], and SNPs in the LPA gene explain much about the variability in the $\operatorname{LP}(\mathrm{a})$ concentration $[16,17]$.

$L P A$ rs3798220 and rs10455872 are extensively studied SNPs in this gene; however, their association with CAD vanished when adjusted for LP(a) levels [8]. These SNPs are present in one of the haplotype blocks of the gene located towards the $5^{\prime}$ end. The nonsynonymous missense polymorphism rs3798220 substitutes methionine in place of isoleucine at position 4399 (Ile4399Me) and causes an increase in $\mathrm{LP}(\mathrm{a})$ concentration $[10,18,19]$. The rs 10455872 is an intronic variant, $49 \mathrm{~kb}$ apart from rs3798220, and both are not in LD with each other. These SNPs may not be directly causal but tagging KIV-2 repeat polymorphism elsewhere in the gene, hence leading to protein size polymorphism [20]. Pakistani population represents a unique ethnic group with distinct genetic architecture [21]. Genetic markers identified in other population need to be validated in this ethnic group before establishing any management and treatment modality based on genetic factors. We, therefore, in the current study, investigated the two LPA SNPs in Pakistani CAD cases and controls to check their association with CAD.

\section{Materials and Methods}

The study comprised of 405 diagnosed CAD cases and 220 healthy controls. The criteria for the selection of study subjects have been described previously [1, 22-25]. The CAD cases were recruited from tertiary care hospitals in Lahore during February 2012 to June 2013. The selected subjects had suffered from a nonfatal myocardial infarction with the diagnosis made by the consultant cardiologists (from various hospitals including Ittefaq Hospital Trust, Jinnah Hospital, Mayo Hospital, and Doctors hospital) based on the reports of ECG, cardiac echo, angiography, troponine T/I, and clinical history. The inclusion criteria for CAD cases were that the subjects were recently diagnosed and had not started lipidlowering or antihypertensive drug therapy. The inclusion criteria for controls were that the subjects were apparently healthy, not having any history of early CAD in the family. The cases and controls represented all socioeconomic groups. Subjects with obesity (BMI $>26 \mathrm{~kg} / \mathrm{m}^{2}$ for Asian populations as described previously [26]) were excluded from the study to reduce the possible confounders but not those with type 2 diabetes because the number of CAD subjects with type 2 diabetes was high and the sample size would have become too small to have adequate power. Subjects seropositive for infectious diseases (HBV, HCV, and HIV) were excluded. All participants gave a written informed consent. The study was approved by the Ethics Committee, University of the Punjab, Lahore, and all procedures were in compliance with the Helsinki declaration.

2.1. Blood Sampling and Biochemical Analyses. Blood samples were taken after $8-12 \mathrm{hr}$ fasting, and half sample was used for DNA isolation, while the rest half was used to obtain serum. Serum was separated by centrifuging gel vacutainers at $10,000 \mathrm{rpm}$ for $10 \mathrm{~min}$, collected in sterilized Eppendorf, and screened for any infectious agents (HBV, HCV, and HIV). Any positive samples were discarded, and safe samples were used for lipid profile determination. Serum total cholesterol (TC), triglycerides (TG), high-density lipoprotein cholesterol (HDL-c), and low-density lipoprotein cholesterol (LDL-c) were measured using commercially available kits (Spectrum Diagnostics, Egypt). Epoch BioTek microplate reader (BioTek Instruments, Highland Park) was used for all optical density measurements.

2.2. Genotyping. The DNA was extracted from whole blood leucocytes using Wizard genomic DNA purification Kit (Promega, USA). The DNA samples were quantified using nanodrop (ND-8000, USA). The concentration of DNA samples was standardized to $1.25 \mathrm{ng} / \mu \mathrm{l}$. The genotyping was carried out in specially designed 384-well plates (Micro Amp). The DNA samples were arrayed into plates by an automated robotic liquid handling system (Biomerk-FX, Beckman Coulter). The quality check of genotyping techniques was maintained by the inclusion of nontemplate controls (NTCs). There were 16 NTCs in each plate of 384 wells. Only those runs were included in the analysis where none of the NTCs crossed the amplification cutoff line. Only the samples which were clearly clustered towards their respective axes were included in the subsequent assay. Both the SNPs were genotyped by TaqMan technique. Because the risk alleles of both SNPs were very low in frequency in our samples, the genotypes were also randomly confirmed by direct DNA sequencing.

2.3. Statistical Analysis. The results were analyzed using Statistical Package for Social Sciences (SPSS) IBM, version 22. Continuous variables like blood lipid levels were compared between cases and controls using an independent sample Student $t$-test. Hardy Weinberg equilibrium was assessed by a $\chi^{2}$ goodness of fit test. For low-risk allele frequency, we used Fisher's exact test for comparing allele frequencies between cases and controls rather than using a chi-square test. Since CAD is a binary variable, the association of the SNPs with CAD was examined using binary logistic regression. The CAD status was considered a dependent variable for different analyses.

\section{Results}

3.1. Study Subjects' Characteristics. The baseline biochemical and anthropometric characteristics of the subjects under study are given in Table 1 . The cases were more diabetic and hypertensive; smoking rate was also higher in cases than controls. Total cholesterol (TC), triglycerides (TG), and LDL-C were significantly higher, whereas HDL-C was lower in cases than controls. Body mass index (BMI) did not differ significantly between the cases and controls.

3.2. LPA Variants and CAD. The features of the SNPs under study are given in Supplementary Table 1. Both SNPs gave good call rates and neither differed significantly from Hardy 
TABle 1: Anthropometric and biochemical parameters of study subjects.

\begin{tabular}{lccc}
\hline Variables & Cases & Controls & $p$ value \\
\hline Number & 405 & 220 & \\
Age (years) & $59.1 \pm 12.6$ & $56 \pm 10.5$ & 0.002 \\
Sex & & & \\
$\quad$ Males $(n)$ & 216 & 120 & 0.27 \\
$\quad$ Females $(n)$ & 189 & 100 & \\
BMI $\left(\mathrm{kg} / \mathrm{m}^{2}\right)$ & $22.46 \pm 6.75$ & $21.46 \pm 9.11$ & 0.119 \\
Diabetes $(\%)$ & 64.6 & 13.6 & $5.1 \times 10^{-34}$ \\
Hypertension $(\%)$ & 62.1 & 16.4 & $8.9 \times 10^{-28}$ \\
Smoking $(\%)$ & 29.5 & 10.5 & $7.3 \times 10^{-08}$ \\
Total cholesterol & $207.5 \pm 53.7$ & $175.4 \pm 43$ & $8.8 \times 10^{-14}$ \\
Triglycerides & $212.4 \pm 70$ & $188 \pm 66.3$ & $2.6 \times 10^{-5}$ \\
LDL-C & $106 \pm 28.9$ & $84.7 \pm 17$ & $6.3 \times 10^{-22}$ \\
HDL-C & $45.2 \pm 11.9$ & $67.4 \pm 16.3$ & $1.8 \times 10^{-66}$ \\
\hline
\end{tabular}

Weinberg equilibrium. The genotyping results showing a clear separation between the common homozygotes and heterozygotes of rs3798220 are shown (supplementary Figures 1 and 2). There was not even a single homozygous for the risk allele observed for this SNP in the subjects under study. For the SNP rs10455872, there was 1 homozygous form of the risk allele and distinct clusters were present for common homozygotes and heterozygotes. The direct DNA sequencing results for common homozygotes and risk heterozygotes for both SNPs are shown in supplementary figures.

For rs 3798220 , the risk allele (C) frequency was 0.005 in cases and 0.002 in controls. While for rs 10455872 , the risk allele $(G)$ frequency was 0.017 in cases and 0.014 in controls. The risk allele frequencies were not significantly different between cases and controls as shown by Fisher's exact test $p$ value (Table 2). The SNPs were not associated with CAD in the studied subjects as shown by their CAD OR $p$ values (Table 3 ).

\section{Discussion}

CAD is the leading cause of death globally despite the measures being taken. The management of heart diseases poses a huge health care burden. Therefore, if the CAD lifetime risk of an individual can be calculated either using genetic markers or serum biomarkers, this can lead to significant reduction of health care burden. The risk score analysis based on genetic markers is routinely done in the developed countries along with Framingham risk score, and some preliminary data is also available for Pakistani $[1,27]$. The use of serum biomarkers (left ventricular ejection fraction determination) has also proved useful; however, in most of the cases, the symptoms begin after the disease has already progressed to an advanced stage $[25,28]$.

In this study, the association of two LPA gene polymorphisms, rs3798220 and rs10455872, with CAD has been studied in Pakistani population. The risk alleles were very low in
TABLE 2: Comparison of RAFs between cases and controls.

\begin{tabular}{lcccccc}
\hline \multirow{2}{*}{ SNP } & \multicolumn{1}{c}{ RAFs } \\
& Alleles & Risk allele & Cases & Controls & ${ }^{*} p$ value & Total \\
\hline rs3798220 & T/C & C & 0.005 & 0.002 & 0.66 & 0.004 \\
rs10455872 & A/G & G & 0.017 & 0.014 & 0.81 & 0.016 \\
\hline
\end{tabular}

${ }^{*}$ Fisher's exact test $p$ value.

TABLE 3: CAD OR of the studied SNPs.

\begin{tabular}{lccc}
\hline SNP & OR & $95 \%$ CI & $p$ value \\
\hline rs3798220 & 2.2 & $0.24-19.63$ & 0.49 \\
rs10455872 & 1.25 & $0.49-3.16$ & 0.64 \\
\hline
\end{tabular}

frequency and were not significantly high in cases than in controls. Due to very low-risk allele frequencies in the target population, these SNPs were not adequately powered to establish an association with CAD in Pakistani population.

The association of $L P A$ polymorphisms with CAD varies among ethnicities due to the difference in allele frequencies among different populations. In our studied subjects, the risk allele frequency of $L P A$ rs 3798220 was very low $(0.04 \%)$ due to which the study was not powered to detect a significant increase in outcome despite a 2.18-fold increase in the odds of CAD. However, the association of this SNP with CAD has been reported in Caucasians $[10,18]$. The low-risk allele frequency $(<1 \%)$ of this SNP suggests that it accounts for only minor variations in CAD incidence. Contrary to this, [29] in another study on Brazilian population, the risk allele frequency was pretty high (6.2\%) but this study failed to find the association of the SNP with CAD, whereas, in a prospective study on healthy people, the risk allele was $3.7 \%$ and increased CAD events 2 times [30].

The SNP LPA rs 10455872 had low RAF (0.16\%) and was not associated with CAD risk. However, in a Brazilian study, the risk allele frequency was $6.4 \%$ and the SNP was significantly associated with CAD [29]. So far, in the studies carried out in European Caucasians, the association of LPA polymorphisms with CAD has been established [20], but the association of LPA rs3798220 with CAD was not replicated in a study on Chinese [5]. In another study comparing European Caucasian, South Asians, and Chinese subjects regarding the genetic polymorphisms of $L P A$, it was found that $L P A$ rs 10455872 was only prevalent in Caucasians [15].

\section{Conclusion}

In conclusion, the low-risk allele frequency of the LPA SNPs is an indication that these SNPs have recently emerged and are not evolutionary conserved. These SNPs are ethnicity specific and affect the disease in specific environmental conditions. According to our findings, these two LPA SNPs are not $\mathrm{CAD}$ associated and cannot be used as independent risk factors for CAD in Pakistani population. However, if other LPA polymorphisms or haplotypes are included, enough power may be obtained for genetic markers to be considered independent $\mathrm{CAD}$ risk factors. 


\section{Data Availability}

All data is available with the corresponding author and can be accessed on request.

\section{Conflicts of Interest}

The authors declare that there are no conflicts of interest regarding the publication of this paper.

\section{Authors' Contributions}

SUS and NS conceived the study concept. SUS carried out the bench work. SUS and NS analyzed the results and drafted the manuscript. SH critically reviewed the manuscript and supervised the study.

\section{Acknowledgments}

Higher Education Commission of Pakistan is acknowledged for providing financial support to the study. Dr. Jackie A Cooper and Ms. Kawah Li are acknowledged for statistical and technical assistance.

\section{Supplementary Materials}

Supplementary Table 1: basic features of SNPs under study. Supplementary Figure 1(a): genotyping results of rs3798220 for cases. Supplementary Figure 1(b): sequencing results of rs3798220. Supplementary Figure 2(a): genotyping results of rs 10455872 for cases. Supplementary Figure 2(b): sequencing results of rs10455872. (Supplementary Materials)

\section{References}

[1] S. U. Shahid, Shabana, J. A. Cooper et al., "Genetic risk analysis of coronary artery disease in Pakistani subjects using a genetic risk score of 21 variants," Atherosclerosis, vol. 258, pp. 1-7, 2017.

[2] G. Isola, A. Polizzi, A. Alibrandi, R. C. Williams, and R. Leonardi, "Independent impact of periodontitis and cardiovascular disease on elevated soluble urokinase-type plasminogen activator receptor (suPAR) levels," Journal of Periodontology, 2020.

[3] G. Isola, A. Lo Giudice, A. Polizzi, A. Alibrandi, P. Murabito, and F. Indelicato, "Identification of the different salivary interleukin- 6 profiles in patients with periodontitis: a crosssectional study," Archives of Oral Biology, vol. 122, 2021.

[4] G. Isola, A. Polizzi, A. Alibrandi, R. C. Williams, and A. Lo Giudice, "Analysis of galectin-3 levels as a source of coronary heart disease risk during periodontitis," Journal of Periodontal Research, vol. 56, no. 3, pp. 597-605, 2021.

[5] Y. Li, M. Luke, D. Shiffman, and J. Devlin, "Genetic variants in the apolipoprotein (a) gene and coronary heart disease," Circulation: Cardiovascular Genetics, vol. 4, no. 5, pp. 565-573, 2011.

[6] H. Kraft, H. Menzel, F. Hoppichler, W. Vogel, and G. Utermann, "Changes of genetic apolipoprotein phenotypes caused by liver transplantation. Implications for apolipoprotein synthesis," Journal of Clinical Investigation, vol. 83, no. 1, pp. 137-142, 1989.
[7] M. L. Koschinsky and S. M. Marcovina, "Structure-function relationships in apolipoprotein (a): insights into lipoprotein (a) assembly and pathogenicity," Current Opinion in Lipidology, vol. 15, no. 2, pp. 167-174, 2004.

[8] J. C. Hopewell, R. Clarke, S. Parish et al., "Lipoprotein (a) genetic variants associated with coronary and peripheral vascular disease but not with stroke risk in the Heart Protection Study," Circulation: Cardiovascular Genetics, vol. 4, no. 1, pp. 68-73, 2011.

[9] H. Schunkert, I. R. König, S. Kathiresan et al., "Large-scale association analysis identifies 13 new susceptibility loci for coronary artery disease," Nature Genetics, vol. 43, no. 4, pp. 333338, 2011.

[10] D. Shiffman, J. P. Kane, J. Z. Louie et al., "Analysis of 17,576 potentially functional SNPs in three case-control studies of myocardial infarction," PLoS One, vol. 3, no. 8, article e2895, 2008.

[11] J. W. McLean, J. E. Tomlinson, W.-J. Kuang et al., "cDNA sequence of human apolipoprotein(a) is homologous to plasminogen," Nature, vol. 330, no. 6144, pp. 132-137, 1987.

[12] R. M. Lawn, K. Schwartz, and L. Patthy, "Convergent evolution of apolipoprotein (a) in primates and hedgehog," Proceedings of the National Academy of Sciences, vol. 94, no. 22, pp. 11992-11997, 1997.

[13] J. E. Tomlinson, J. W. McLean, and R. M. Lawn, "Rhesus monkey apolipoprotein(a): sequence, evolution, and sites of synthesis," The Journal of Biological Chemistry, vol. 264, no. 10 , pp. 5957-5965, 1989.

[14] H. Dieplinger and G. Utermann, "The seventh myth of lipoprotein(a)," Current Opinion in Lipidology, vol. 10, no. 3, pp. 275-284, 1999.

[15] M. B. Lanktree, S. S. Anand, S. Yusuf, R. A. Hegele, and SHARE Investigators, "Comprehensive analysis of genomic variation in the LPA locus and its relationship to plasma lipoprotein (a) in South Asians, Chinese, and European Caucasians," Circulation: Cardiovascular Genetics, vol. 3, no. 1, pp. 39-46, 2010.

[16] J. Chretien, J. Coresh, Y. Berthier-Schaad et al., “Three singlenucleotide polymorphisms in LPA account for most of the increase in lipoprotein (a) level elevation in African Americans compared with European Americans," Journal of Medical Genetics, vol. 43, no. 12, pp. 917-923, 2006.

[17] R. C. Deo, J. G. Wilson, C. Xing et al., "Single-nucleotide polymorphisms in LPA explain most of the ancestry-specific variation in Lp (a) levels in African Americans," PLoS One, vol. 6, no. 1, article e14581, 2011.

[18] M. M. Luke, J. P. Kane, D. M. Liu et al., "A polymorphism in the protease-like domain of apolipoprotein (a) is associated with severe coronary artery disease," Arteriosclerosis, Thrombosis, and Vascular Biology, vol. 27, no. 9, pp. 2030-2036, 2007.

[19] W. Lu, Y.-C. Cheng, K. Chen et al., "Evidence for several independent genetic variants affecting lipoprotein (a) cholesterol levels," Human Molecular Genetics, vol. 24, no. 8, pp. 23902400, 2015.

[20] R. Clarke, J. F. Peden, J. C. Hopewell et al., "Genetic variants associated with Lp (a) lipoprotein level and coronary disease," The New England Journal of Medicine, vol. 361, no. 26, pp. 2518-2528, 2009.

[21] N. Shabana, S. Ashiq, A. Ijaz et al., "Genetic risk score (GRS) constructed from polymorphisms in the PON1, IL-6, ITGB3, and $\mathrm{ALDH} 2$ genes is associated with the risk of coronary artery 
disease in Pakistani subjects," Lipids in Health and Disease, vol. 17, no. 1, p. 224, 2018.

[22] S. U. Shahid, J. A. Cooper, A. Rehman, and S. E. Humphries, "Association of ACE and NOS3 gene polymorphism with blood pressure in a case control study of coronary artery disease in Punjab, Pakistan," Pakistan Journal of Zoology, vol. 48 , no. $4,2016$.

[23] S. U. Shahid, J. A. Cooper, A. Rehman, and S. E. Humphries, "Association of ACE and NOS3 gene polymorphisms with blood pressure in a case control study of coronary artery disease in Punjab, Pakistan," Pakistan Journal of Zoology, vol. 48, no. 4, pp. 1125-1132, 2016.

[24] S. U. Shahid, Shabana, A. Rehman, and S. Hasnain, "Role of a common variant of fat mass and obesity associated (FTO) gene in obesity and coronary artery disease in subjects from Punjab, Pakistan: a case control study," Lipids in Health and Disease, vol. 15, no. 1, 2016.

[25] S. U. Shahid, N. Shabana, A. Rehman, and S. Humphries, "GWAS implicated risk variants in different genes contribute additively to increase the risk of coronary artery disease (CAD) in the Pakistani subjects," Lipids in Health and Disease, vol. 17, no. 1, p. 89, 2018.

[26] S. U. S. Shabana, S. Ullah Shahid, K. Wah Li et al., "Effect of six type II diabetes susceptibility loci and an FTO variant on obesity in Pakistani subjects," European Journal of Human Genetics, vol. 24, no. 6, pp. 903-910, 2016.

[27] S. U. Shahid, S. , J. A. Cooper et al., "Effect of SORT1, APOB and APOE polymorphisms on LDL-C and coronary heart disease in Pakistani subjects and their comparison with Northwick Park Heart Study II," Lipids in Health and Disease, vol. 15 , no. $1,2016$.

[28] E. Una Cidon, T. Hickish, and P. Alonso, "The role of cardiac biomarkers in early detection of cardiotoxicity in breast cancer treated with trastuzumab in the adjuvant setting: a systematic review," Open Science Journal, vol. 6, no. 1, 2021.

[29] B. C. Santos, C. T. Bueno, P. A. Lemos, J. E. Krieger, and A. C. Pereira, "LPA rs10455872 polymorphism is associated with coronary lesions in Brazilian patients submitted to coronary angiography," Lipids in Health and Disease, vol. 13, no. 1, 2014.

[30] D. I. Chasman, D. Shiffman, R. Y. Zee et al., "Polymorphism in the apolipoprotein(a) gene, plasma lipoprotein(a), cardiovascular disease, and low-dose aspirin therapy," Atherosclerosis, vol. 203, no. 2, pp. 371-376, 2009. 